\title{
DKA precipitated by iatrogenic thyrotoxicosis
}

\begin{abstract}
28 year old compliant man with a history of type I diabetes mellitus and primary hypothyroidism, presented to the ER for a two day history of fatigue, nausea, vomiting and abdominal pain. On physical exam, patient was drowsy with a dry skin and normal temperature. Kussmaul respirations were noted and HR was $120 \mathrm{bpm}$. Laboratory workup showed glucose of 747, positive serum acetone, creatinine 4, and metabolic acidosis with anion gap of 64. ABG revealed the following: $\mathrm{pH}$ 7.071, $\mathrm{PaO}_{2} 117.3, \mathrm{PaCO}_{2} 20$, and $\mathrm{HCO}_{3} 5.8 \mathrm{meq} / \mathrm{L}$. Appropriate treatment for DKA was initiated and despite adequate fluid resuscitation, patient remained tachycardic. EKG showed sinus tachycardia. Upon further questioning the patient, he reported those 2 weeks prior, he started experiencing erectile dysfunction and decided on his own to increase his dose of levothyroxine from 300 to 600 mcg per day. Thyroid panel was ordered and it showed a TSH of 0.014 and FT4 of 3.1 so beta blocker was started. Twelve hours later, his DKA resolved as well as his acute renal failure and he became hemodynamically stable.
\end{abstract}

Conclusion: Thyroid storm and DKA are both potentially fatal especially that DKA may obscure the typical clinical presentation of thyrotoxicosis. Prognosis varies depending on whether or not these conditions are detected early and treated sufficiently. Persistent tachycardia following correction of dehydration in aseptic patients with DKA should raise the possibility of thyrotoxicosis especially for those known to have a thyroid disease. Diabetic patients taking thyroid hormones should be made aware of the complications that may result from excess doses.

Keywords: diabetic ketoacidosis, graves' disease, hypothyroidism, free fatty acid, thyroid stimulating hormone, thyrotoxicosis, diabetes mellitus type 1 , hyperglycemia, growth hormone, blood pressure
Volume 2 Issue 4 - 2015

\author{
Layal Esper,' Pierre El Hachem, ${ }^{2}$ Alice \\ Chedid,' Carla Sawan, ${ }^{3}$ Karelene Willaims ${ }^{1}$ \\ 'Department of Internal Medicine, Englewood Hospital and \\ Medical Center, USA \\ ${ }^{2}$ Department of Palliative Medicine, Cleveland Clinic, USA \\ ${ }^{3}$ Department of Endocrinology, University of Balamand, Lebanon
}

Correspondence: Layal Esper, Department of Internal Medicine, Englewood Hospital and Medical Center 276 Engle St Apt I5B, Englewood, New Jersey, USA, Tel (201)6373925, Email layalesper@gmail.com

Received: October 05, 2015 | Published: October 19, 2015
Abbreviations: DKA, diabetic ketoacidosis; ABG, arterial blood gas; TSH, thyroid-stimulating hormone; GLUT2, glucose transporter 2; FFA, free fatty acid; GH, growth hormone

\section{Introduction}

Thyrotoxicosis, whether caused by Graves' disease, toxic adenoma or toxic multinodular goiter, has been reported as a precipitating factor for diabetic ketoacidosis in patients with Diabetes mellitus type 1. We are describing in this report a rare case of DKA triggered by excessive thyroid hormone intake.

\section{Case description}

Our patient is a 28 year old man with a history of type I diabetes mellitus and primary hypothyroidism diagnosed since childhood. He presented to the ER with a two days history of fatigue, nausea, vomiting and abdominal pain. No recent illnesses, ingestions, or skipped doses of insulin. No obvious precipitating factors. On physical exam, the patient was drowsy and his physical exam was remarkable for dry skin and mucous membranes. We noted Kussmaul's breathing (what was the respiratory rate??), Blood pressure was XXX and heart rate was 120 beats per minute. Laboratory workup showed a glucose level of $747 \mathrm{mg} / \mathrm{dL}$, positive serum acetone, and creatinine level of $4 \mathrm{mg} /$ $\mathrm{dL}$, and metabolic acidosis with an anion gap of 64. An arterial blood gas showed the following: $\mathrm{pH} 7.071, \mathrm{PaO}_{2}$ 117.3, $\mathrm{PaCO}_{2} 20$, and $\mathrm{HCO}_{3} 5.8 \mathrm{meq} / 1$. Appropriate treatment for DKA was initiated and despite adequate fluid resuscitation and resolution of the DKA, the patient remained tachycardic with an ECG showing sinus tachycardia.
Upon further questioning of the patient, he reported those 2 weeks prior to presentation, he started experiencing erectile dysfunction and increased his dose of levothyroxine from $300 \mathrm{mcg}$ to $600 \mathrm{mcg}$ daily on his own. Thyroid panel showed a TSH level of $0.014 \mathrm{mIU} / \mathrm{L}$ and FT4 level of $3.1 \mathrm{ng} / \mathrm{L}$ (normal range being 0.8-1.8 ng/L). A beta blocker was started with watchful monitoring of the patient's condition. Twelve hours later, he became hemodynamically stable and regained normal kidney function and normal anion gap.

\section{Discussion}

DKA may obscure thyrotoxicosis and/or infection, resulting in a fatal outcome. This case emphasizes that the possibility of thyrotoxic storm or hyperthyroidism should be considered as soon as possible, even when the symptoms are not so obvious in patients with DKA. Detailed history and physical exam may help diagnose hyperthyroidism; however it may sometimes be obscured. In our case the presence of hyperthyroidism was suspected early although masked because of history of noncompliance, and tachycardia which didn't improve with aggressive hydration. Thyroid hormones affect glucose metabolism via several mechanisms. Hyperthyroidism has been recognized to enhance hyperglycemia. ${ }^{1}$ One mechanism is the decreased half-life of insulin, likely due to the increased the rate of degradation and enhanced release of inactive insulin precursors. ${ }^{2,3}$ In addition, untreated hyperthyroidism was associated with a reduced C-peptide to proinsulin ratio suggesting an underlying defect in proinsulin processing. ${ }^{4}$ Another mechanism explaining the relationship is the increase in glucose gut absorption mediated by the excess thyroid hormones. ${ }^{5,6}$ 
Hyperglycemia is also enhanced in hyperthyroidism via several mechanisms. Thyroid hormones produce an increase in the hepatocyte plasma membrane concentrations of GLUT2 which is the main glucose transporter in the liver, and consequently, contribute to the increased hepatic glucose output and abnormal glucose metabolism. ${ }^{7,8}$ Additionally, increased lipolysis is observed in hyperthyroidism resulting in an increase in FFA that stimulates hepatic gluconeogenesis. The increased release of FFA could partially be explained by an enhanced catecholamine-stimulated lipolysis induced by the excess thyroid hormones. ${ }^{9}$ Moreover, the non-oxidative glucose disposal in hyperthyroidism is enhanced resulting in an overproduction of lactate that enters the Cori cycle and promotes further hepatic gluconeogenesis. The increase in GH, glucagon and catecholamine levels associated with hyperthyroidism further contributes to the impaired glucose tolerance. ${ }^{10-12}$

It is well known that diabetic patients with hyperthyroidism experience worsening of their glycemic control and thyrotoxicosis has been shown to precipitate diabetic ketoacidosis in subjects with diabetes. ${ }^{13,14}$ Besides all of the above described mechanisms, thyroid hormones can indirectly affect glucose metabolism through modulation of energy homeostasis. Although the underlying mechanisms have not yet been clearly defined, thyroid hormones have been shown to alter the expression of uncoupling proteins in brown adipose tissue involved in effective thermoregulation. ${ }^{15}$ Herein we presented the case of exogenous hyperthyroidism precipitating and being masked by DKA. It is essential to bear in mind the overlap and of both diseases and to investigate hyperthyroidism in cases of DKA where suspicion is warranted.

\section{Conclusion}

Thyrotoxicosis and DKA are both potentially fatal especially that DKA may obscure the typical clinical presentation of thyrotoxicosis. Thyroid hormone affects glucose metabolism via several mechanisms. It decreases half-life of insulin, reduces the C-peptide/proinsulin ratio, and increases plasma concentration via intestinal absorption and hepatic gluconeogenesis. Prognosis is dependent on early diagnosis and appropriate timing of treatment and intervention. Auto-immune thyroid disorders are the most common disorders found in patients with type 1 diabetes mellitus, both hypothyroidism and hyperthyroidism. Persistent tachycardia following correction of dehydration in aseptic patients with DKA should raise the possibility of thyrotoxicosis especially for those known to have a thyroid disease. Diabetic patients taking thyroid hormones should be made aware of the complications that may result from excess doses.

\section{Acknowledgments}

None.

\section{Conflicts of interest}

The auhtor declares there is no conflicts of interest.

\section{References}

1. Maxon HR, Kreines KW, Goldsmith RE, et al. Long-term observations of glucose tolerance in thyrotoxic patients. Arch Intern Med. 1975;135(11):1477-1480.

2. O'Meara NM, Blackman JD, Sturis J, et al. Alterations in the kinetics of C-peptide and insulin secretion in hyperthyroidism. J Clin Endocrinol Metab. 1993;76(1):79-84.

3. Dimitriadis G, Baker B, Marsh H, et al. Effect of thyroid hormone excess on action, secretion, and metabolism of insulin in humans. Am J Physiol. 1985;248(5):E593-E601.

4. Beer SF, Parr JH, Temple RC, et al. The effect of thyroid disease on proinsulin and C-peptide levels. Clin Endocrinol (Oxf). 1989;30(4):379-383.

5. Levin RJ, Smyth DH. The effect of the thyroid gland on intestinal absorption of hexoses. J Physiol. 1963;169(4):755-769

6. Matty AJ, Seshadri B. Effect of thyroxine on the isolated rat intestine. Gut. 1965;6:200-202.

7. Kemp HF, Hundal HS, Taylor PM. Glucose transport correlates with GLUT2 abundance in rat liver during altered thyroid status. Mol Cell Endocrinol. 1997;28(1-2):97-102.

8. Mokuno T, Uchimura K, Hayashi R, et al. Glucose transporter 2 concentrations in hyper- and hypothyroid rat livers. $J$ Endocrinol. 1999;160(2):285-289.

9. Vaughan M. An in vitro effect of triiodothyronine on rat adipose tissue. J Clin Invest. 19667;46(9):1482-1491.

10. Miki N, Ono M, Hizuka N, et al. Thyroid hormone modulation of the hypothalamic growth hormone $(\mathrm{GH})$ - releasing factor-pituitary $\mathrm{GH}$ axis in the rat. J Clin Invest. 1992;90(1):113-120.

11. Tosi F, Moghetti P, Castello R, et al. Early changes in plasma glucagon and growth hormone response to oral glucose in experimental hyperthyroidism. Metabolism. 1996;45(8):1029-1033.

12. Sestoft L,Christensen NJ, Saltin B. Responses of glucose and glucoregulatory hormones to exercise in thyrotoxic and myxoedematous patients before and after 3 months of treatment. Clin Sci (Lond). 1991;81(1):9199.

13. Sola E, Morillas C, Garzon S, et al. Association between diabetic ketoacidosis and thyrotoxicosis. Acta Diabetol. 2002;39(4):235-237.

14. Bhattacharyya A, Wiles PG. Diabetic ketoacidosis precipitated by thyrotoxicosis. Postgrad Med J. 1999;75( 883):291-292.

15. Nagasaki T, Inaba M, Hiura Y, et al. Plasma levels of adiponectin and soluble thrombomodulin in hypothyroid patients with normal thyroid function following levothyroxine replacement therapy. Biomed Pharmacother. 2005;59(10):571-577. 